\title{
LOS ESTUDIOS DE SOCIOLOGÍA URBANA EN AMÉRICA LATINA, UN ESTADO DEL ARTE. LA SITUACIÓN EN ARGENTINA. 1957-1976
}

\author{
Dra Verónica Paiva \\ Cátedra "Sociología Urbana"- CIHAM, Facultad de Arquitectura, Universidad de Buenos Aires \\ vtpaiva@gmail.com
}

\section{RESUMEN}

El artículo aborda el surgimiento de la sociología urbana en Argentina entre 1957 y 1976 . Se estudian los centros relacionados con los estudios urbanos, los profesionales que estuvieron a cargo de la materia sociología urbana y el perfil teórico con que impartieron la disciplina. En la medida de lo posible, se sitúan las instituciones y las corrientes teóricas con el respectivo escenario teórico, social y político del país. El período comprende los años 1957 a 1976, es decir, desde el nacimiento formal de la carrera de Sociología a partir de la labor de Gino Germani y el golpe de estado de 1976 y su influencia en el cierre de varias instituciones relacionados con los estudios urbanos. Fue realizado con entrevistas en profundidad, memorias institucionales y lectura de textos clave de ese período con metodología acorde al tratamiento de ese tipo de fuentes, como el análisis de entrevistas y de contenido.

Palabras clave: Sociología Urbana- Estudios Urbanos en Argentina - Sociología Argentina

\section{ABSTRACT}

The article deals with the emergence of urban sociology in Argentina between 1957 and 1976. Studies related to urban studies, professionals who were in charge of urban sociology and the theoretical profile with which they taught the discipline are studied. As far as possible, institutions and theoretical currents are placed with the respective theoretical, social and political scenario of the country. The period covers the profile of the studies carried out between 1957 and 1976, that is, from the formal birth of the Sociology career from the work of Gino Germani and the 1976 coup d'état and its influence on the closure of several institutions related to urban studies. It was carried out with in-depth interviews, institutional memories and reading of key texts of that period with methodology according to the treatment of that type of sources, such as the analysis of interviews and content. 


\section{Introducción}

La ponencia surge a partir de mi labor como responsable de la materia Sociología Urbana en la carrera de Arquitectura de la Universidad de Buenos Aires. Dado que durante la materia se explica el recorrido histórico de la sociología urbana a nivel internacional, surgió el interrogante sobre los inicios de dicha rama de la sociología en Argentina y quedó clara la inexistencia de estudios sobre este asunto. En ese contexto se inicia la investigación que subyace a esta ponencia, la que se encuentra en sus inicios.

Antes de comenzar el artículo conviene aclarar que si bien los inicios de la sociología urbana y de los estudios urbanos en el país, crecieron y se gestaron juntos y en ocasiones se confunde la perspectiva de uno y otro, en este trabajo nos centraremos específicamente en el surgimiento de la sociología urbana. Entenderemos por ésta a la disciplina que estudia los procesos sociales inscriptos en el espacio urbano (Saunders, 1986) y a los estudios urbanos como un conjunto de disciplinas -dentro de las cuales se encuentra la sociología - destinadas a pensar el fenómeno desde diferentes marcos analíticos en vistas a la planificación de las ciudades.

Para elaborar el trabajo recurrí a bibliografía secundaria, en especial la referida a los estudios urbanos en América Latina y los pocos que existen sobre Argentina, tomé entrevistas a profesionales clave en los inicios de los estudios urbanos a nivel local y recurrí a memorias institucionales de centros de investigación que fueron importantes en el despliegue de los estudios urbanos.

A partir del análisis de todo ese material, pude delinear la existencia de tres períodos en el avance de la disciplina. El primero de ellos desde el inicio de la carrera por Gino Germani en 1958, en el cual dicho sociólogo produce investigaciones que son clave para entender la perspectiva sociológica sobre la ciudad y la urbanización hacia fines de los años '50. Un segundo período signado por la creación del Instituto de Planeamiento Urbano y Regional (IPRUL) en Rosario en donde surge una cátedra llamada Sociología Urbana bajo la perspectiva de la ecología humana y un tercer momento, en 1966, en donde se crea el Centro de Estudios Urbanos y Regionales (CEUR) en donde se forman sociólogos que fueron importantes en el avance de la disciplina y que incorporan la perspectiva del marxismo estructuralista de la sociología francesa, vigentes por aquella etapa. Marco el final del recorrido en 1976 porque en Argentina se produce un golpe de estado que señala el exilio de muchos investigadores y el ocaso de algunas teorías esencialmente el marxismo estructuralista - que convivirán con nuevas perspectivas teóricas cuando resurjan los estudios académicos en 1983, con el advenimiento de la democracia.

De acuerdo con ello, esta ponencia se dividirá en los siguientes puntos. En primera instancia realizaré un breve estado de la cuestión sobre los estudios urbanos y la sociología urbana en América Latina y Argentina y luego tres apartados dedicados a los distintos períodos que señalé. Hacia el final elaboraré algunas conclusiones preliminares.

Como esbocé al inicio, se trata de una investigación que se encuentra en sus comienzos y de cuyo avance se acompañan los resultados preliminares.

\section{Estudios urbanos y de sociología urbana en América Latina y Argentina. Un estado de la cuestión.}

Mientras a nivel internacional existen muchas investigaciones que han tratado sobre el desarrollo de la sociología urbana como las de Topalov (2012) o Ullán de Rosa (2014), hay menor cantidad de estudios que hayan abordado el caso para América Latina y muchos menos para Argentina. En cambio, es mayor la cantidad de trabajos focalizados en el desarrollo de los estudios urbanos en Latinoamérica y en Argentina.

Sobre América Latina puede citarse el estudio de Martha Schteingart realizado por tres equipos de trabajo que dieron cuenta del estado de los estudios urbanos en diferentes regiones: por un lado, México, Colombia y Centroamérica, por otro, Brasil y Venezuela y un tercer grupo formado por los países del Cono Sur más Perú, Bolivia y Ecuador. El trabajo concluye que -en el primer grupo- la investigación urbana se desarrolló sistemáticamente durante los años 1970, sin perjuicio de que en México hubo estudios aislados en las décadas de 1940, 1950 y 1960 al igual que en Colombia. De modo similar, la expansión e institucionalización de los estudios urbanos en Brasil y Venezuela se produjo en la década de 1960 por el apoyo del estado para la generación de instituciones de investigación y posgrados especializados en esta problemática. Ese mismo cuadro de situación surgió en las investigaciones relativas a los países del Cono Sur como Uruguay y Argentina. (Schteingart, 2000). 
Sobre los estudios urbanos en América Latina también puede citarse el trabajo de Gorelik relativo al debate antropológico sobre las características del continuum rural urbano que se produjo entre Robert Redfield y Oscar Lewis en Norteamérica hacia la década de 1950 y el impacto de este debate en el ámbito latinoamericano. Respecto de este asunto, Gorelik aborda el modo en que se retoma el tema en Lima, Río de Janeiro y Buenos Aires, en este último caso analizando una investigación de Germani sobre la Isla Maciel que presenta en un Seminario sobre problemas de urbanización en América Latina organizado por la UNESCO en 1959, en donde trata de analizar qué aspectos se cumplen y cuáles no de la transición rural -urbana. (Gorelik, 2008). Siempre a nivel latinoamericano cabe citar el interesante estudio de Oscar Yujnovsky sobre los enfoques teórico ideológico de los estudios urbanos entre las décadas de 1950 y 1970 en donde propone la existencia de tres períodos: uno primero en la década de 1950 en donde se realizan estudios atravesados por la teoría del desarrollo a partir de la CEPAL, con un enfoque macro y economicista centrado en las posibilidades de desarrollo de una región, de un país o grupo de países. Estos estudios tuvieron impacto en la elaboración de planes de desarrollo que incluían una dimensión espacial y de planificación urbana y regional en las cuales la preocupación principal era la aceleración de la urbanización, las migraciones campo-ciudad y la formación de villas de emergencia y rancheríos que surgían como consecuencia de la industrialización sustitutiva de importaciones. El seminario de 1959 sobre "Problemas de urbanización en América Latina de 1959 (también citado en el texto de Gorelik) ofrece perspectivas urbanas tributarias de esta mirada en donde los estudios sociológicos se centran en las villas y áreas marginales de América Latina y se analizan en términos de "patología", "anomia", "desorganización familiar" y "falta de integración". (Yujnovksy, 1975:7). Siempre según este autor, existe un segundo período situado en la década de 1960 en donde predomina el enfoque de la "modernización" y la urbanización desaparece como fuente de problemas. La ciudad es considerada como manifestación del proceso de cambio o como variable independiente necesaria para el desarrollo y las villas ya no son una "patología". Por último, una nueva etapa se perfila en la década de 1970 en donde a partir de la evidencia del fracaso del modelo desarrollista y de la planificación estatal como vehículo de crecimiento, surgen estudios críticos de perfil marxista o tributarios de la teoría de la dependencia. (Yujnovksy, 1975). Además, cabe citar un trabajo de Emilio Duhau para México y América Latina, en donde aborda las teorías urbanas presentes en las líneas de investigación iniciadas a partir de la década de 1980. (Duhau y otros, 1991)

Por último, entre otra de las investigaciones realizadas sobre los estudios urbanos a nivel general, puede citarse la de Beatriz Cuenya, quien, luego de repasar los paradigmas y perspectivas de la década de 1970, pone el foco en la situación posterior a 1980 y en particular en 1990. Sobre esta etapa la autora señala el rumbo tomado por los estudios urbanos con la incorporación de las investigaciones de Saskia Sassen sobre la globalización económica y sus efectos en el espacio urbano $(1994,1997)$ y las de Donzelot y Jaillet sobre la "nueva cuestión urbana" (1997). Estos autores señalan las diferencias entre lo que fue la "cuestión urbana" durante el siglo XIX y la nueva cuestión urbana en la década de los '90. Mientras la primera era la expresión territorial de la cuestión social y se refería a las condiciones habitacionales de los obreros que estaban integrados socialmente y laboralmente pero no espacialmente, la "nueva cuestión urbana" señala la existencia de espacios urbanos segregados, en donde hay grupos de personas segregadas espacialmente pero también socialmente ya que están desempleadas y sin integración a la sociedad y al espacio general (Cuenya, 2004)

Sobre los estudios urbanos en Argentina, hay un trabajo de Mabel Manzanal que aborda el tema hasta mediados de los '80 con una perspectiva similar a la de Yujnovsky a nivel latinoamericano (Manzanal, 1988) y otro de Nora Clichevsky sobre el estado de los estudios urbanos desde el advenimiento de la democracia en 1983. En ese texto, Clichevsky estudia las instituciones, temáticas y nuevas perspectivas que surgen desde 1983 como el interés por las cuestiones de vivienda popular, el medio ambiente urbano, la vida cotidiana de los sectores populares, las ciudades intermedias y pequeñas y el tema del género y el hábitat urbano. (Clichevsky, 1988). Otro trabajo sobre Argentina es un estado de la cuestión elaborado por Alicia Novick sobre los modos de realizar la historia urbana e historia de la ciudad a través del tiempo, en donde distingue tres etapas: una primera en donde prima la historia como "evolución urbanística" en los estudios que pretendían institucionalizar el urbanismo como ciencia, una segunda, situada luego de la segunda posguerra, signada por los estudios de tendencia y una tercera caracterizada por la elaboración de "historias de la ciudad" que tratan de relacionar ideas y prácticas, en especial las ideas científicas y su relación con la práctica espacial (Novick, 2004). Más reciente es la tesis de Alejandra Monti sobre Jorge Enrique Hardoy y su labor en la iniciación de los estudios urbanos 
en Argentina, sobre las instituciones creadas en esa etapa y sobre las ideas de planificación urbana que se plasmaron en esos centros. (Monti,2015). También sobre el rol de la revista de la Sociedad Interamericana de Planificación (SIAP) en la consolidación de un saber experto en materia de planificación urbana en el período que corre entre 1967 y 1970 (Monti, 2017). Otras investigaciones relacionadas con la expansión de la planificación urbana y regional en América Latina son las que ha llevado adelante Jajamovich sobre el surgimiento de la Comisión de Desarrollo urbano y regional de CLACSO entre 1967 y 1973 y su relación con la planificación para el desarrollo en América Latina (Jajamovich, 2016)

Respecto a los trabajos que tratan el surgimiento de la sociología urbana en Argentina, cabe citar el de Ivana Socoloff sobre el papel de Enrique Pichón Riviére en la elaboración de escritos referidos a las problemáticas de la vida en la ciudad hacia 1950, más tarde la "sociología científica" de Gino Germani y su foco en las consecuencias sociales de la urbanización y, por último, el rol del marxismo estructuralista en los estudios urbanos de Argentina, producidos desde fines de la década de 1960. (Socoloff, 2012). Siempre en relación con los inicios de la sociología urbana en Argentina, pueden citarse los trabajos de Pablo Roffé sobre una investigación de Gino Germani en la Isla Maciel y su visión sobre los efectos de la urbanización y modernización en los sectores obreros (2013) y sobre el mismo trabajo, la investigación de Juan Ignacio Trovero centrada en las herramientas metodológicas utilizadas por Germani. (Trovero, 2017)

Tal como se ha visto, las investigaciones referidas a los estudios urbanos en Argentina son escasas y recientes y mucho más aquellas centradas específicamente en el surgimiento y desarrollo de la sociología urbana. Por lo cual, esta ponencia retomará el conocimiento recibido de las investigaciones previas y sobre esta base profundizará en el desarrollo de algunas instituciones relacionadas con los estudios urbanos en el país, en la formación de los profesionales que estuvieron a cargo de los estudios de sociología en cada período y en el perfil teórico que dieron a sus trabajos. En cuanto sea posible se tratará de ubicar dichas investigaciones en el contexto teórico, social y político de cada etapa.

\section{Germani y la Sociología Urbana}

Tal como es sabido, Gino Germani fue el creador de la la carrera de sociología en la Universidad de Buenos Aires en 1957. Si bien hasta ese momento existieron profesionales de otras disciplinas (médicos, abogados) que realizaron estudios vinculados a la comprensión de la dinámica social argentina o que estuvieron a cargo de "cátedras" de Sociología dentro de la UBA o de la Universidad de La Plata, lo cierto es que la "carrera" se implementa a partir del Decreto Resolución № 527 del 14 de marzo de 1957 bajo la dirección de Gino Germani.

Mucho se ha escrito sobre los estudios de sociología de esta etapa y también sobre la impronta de Germani sobre esos primeros estudios de sociología. Entre otras, se ha repasado el perfil empirista y estadístico con que intentó dotar de cientificidad a la disciplina (Buccafusca, Serulnicoff y Solari, 2000); su proyecto editorial e intelectual, los totalitarismos, la sociedad de masas y el populismo (Blanco, 2006) o su preocupación por el paso de la sociedad tradicional a la sociedad moderna (Stropparo, 2011). Sin embargo, su vinculación con los estudios urbanos y más específicamente, con los inicios de la sociología urbana, ha sido escasamente recorrido y sólo existen los trabajos de Socoloff, Trovero y Roffé que fueron citados en el punto anterior.

¿Existe relación entre la labor científica e institucional de Gino Germani y la expansión de la sociología urbana en Argentina? En este sentido, es posible afirmar que si bien Germani no fue un sociólogo urbano en el sentido de hacer de esa rama su perfil profesional específico, sí tuvo un rol importante en el desarrollo de la sociología urbana en Argentina, debido las temáticas que trabajó en sus investigaciones, su posición en instituciones claves de la época y el conjunto de profesionales relacionados con los estudios urbanos que se formaron en sus equipos. ¿De qué se trata?

Hacia mitad de la década de 1950 Germani dirige una investigación sobre "los efectos sociales de la urbanización en un área obrera del Gran Buenos Aires" en la Isla Maciel. Se trata de un estudio propiciado por el recién creado Instituto de Extensión Universitario de la UBA en 1956 en donde un equipo dirigido por él estudia las condiciones de vivienda, alimentación, recreación, inteligencia y salud de los habitantes de la Isla Maciel, realizado entre 1957 y 1958. Dicho trabajo es llevado al "Seminario sobre problemas de urbanización en América Latina" realizado en 1959 en Santiago de Chile, auspiciado por la UNESCO y la CEPAL que resulta clave en la expansión de las ideas desarrollistas impulsadas por Raúl Prebisch y que tuvo como objetivo demostrar los 
modos y consecuencias de la urbanización y la modernización en los países no centrales. En ese contexto, más de 50 países llevan estudios referidos a los efectos de la urbanización en las condiciones de vida de la población, entre ellos, este trabajo sobre la Isla Maciel dirigido por Germani.

¿Qué perspectiva sobre la urbe, la urbanización y la vida social aparece en dicha investigación? En el trabajo se analizan tres grupos poblacionales: Ios nativos de la Isla, los inmigrantes de larga data en el sitio y los recién inmigrados. Según la investigación, los recién llegados tenían una inserción laboral más débil (changadores y otro tipo de trabajadores portuarios) vivían en la "villa" (y no en la "Isla"), es decir, en un aglomerado con viviendas muy precarias de chapa y cartón que habían autoconstruido y a diferencia de los grupos de mayor antigüedad, entre ellos primaba la desorganización familiar, las adicciones y la vagancia. Según la investigación dirigida por Germani, en el grupo más reciente existían pautas que traían de los lugares de origen, tal como las uniones libres, el número de hijos y el autoritarismo, pero también otras que nacieron o se incrementaron a partir de la inmigración, como el juego o la prostitución. Con lo cual, y a tono con la hipótesis sobre la modernización que se manejaba en el Seminario de 1959, la urbanización en los países latinoamericanos no siempre era signo de modernización por industrialización, si no de mayor urbanización que industrialización y en condiciones de alta precariedad habitacional, laboral y social. La lectura teórica del problema de la migración y la urbanización es realizada en términos de "anomia, desorganización familiar, prostitución y males urbanos" del mismo modo en que la Escuela de Chicago entendió las consecuencias de la migración y la vida urbana en la Chicago de los años 1920. Si se tiene en cuenta que el organizador general del Seminario es Phillips Hauser, por entonces Director del Departamento de Sociología de Chicago, que uno de los asistentes es Herbert Blumer y que Gino Germani asiste como delegado de la UNESCO, la filiación teórica resulta coherente con la perspectiva dominante, aunque, como veremos, Germani tiene una mirada ambivalente sobre el rol de la ciudad sobre la vida poblacional, ya que en otros escritos aparece en términos progresistas y optimistas, ligados a la modernización.

Un texto paradigmático de esta segunda perspectiva es "La ciudad como mecanismo integrador" (Germani, 1967) en donde pone en evidencia el rol de la ciudad en ese sentido y como mecanismo de modernización de las poblaciones. Manifiesta que la integración puede ser geográfica o social. La primera se refiere al desarrollo equilibrado de las distintas regiones o provincias de un país y la segunda es la integración específicamente social. Dicha integración implica tres momentos a) liberación y disponibilidad, b) movilización c) integración a las estructuras modernas. La liberación alude a dejar de vivir según las pautas y modos de conducta de la sociedad tradicional y la disponibilidad es, justamente, quedar disponible para la incorporación de nuevas pautas. Por último, la movilización es la incorporación de las pautas de la sociedad moderna, las que, si bien no son definidas explícitamente por Germani, se refieren a la incorporación en estructuras modernas tales como el trabajo asalariado en fábricas, estilo de familia nuclear, etc. La ciudad es un gran estimulante de la movilización centralmente a través de dos mecanismos: la educación y los medios de comunicación. Estas dos instituciones permiten la liberación de las pautas tradicionales y ponen al sujeto en disponibilidad para la adquisición de nuevos valores, consumo y ocupaciones. Desde esta posición la "marginalidad" es no estar integrado en todo o en parte a estas estructuras modernas y la sociología tiene que estudiar las diferentes formas de marginalidad que existen en un país o región y cómo se conectan. Como vemos, en este texto Germani discute con la Escuela de Sociología Urbana de Chicago que sólo veía la urbanización y la migración campo ciudad, en términos de anomia y desorganización social, es decir, desde una mirada negativa y crítica que más bien revalorizaba la vida rural y comunitaria, sin analizar los mecanismos de integración y modernización que produce la ciudad.

En relación con el papel de Germani en las instituciones ligadas al desarrollo de la sociología urbana, no sólo participa en el seminario de Chile con un trabajo sobre los efectos de la urbanización en la población, si no que al crear la carrera de sociología de la UBA de 1957 genera un seminario sobre "Seminario de Migraciones y Vivienda Popular", muy cercano a la temática urbana. Por otro lado, la carrera de esa etapa otorgaba un "Certificado de Estudios Sociológicos" para graduados universitarios provenientes de otras carreras que cursaron algunos arquitectos que son centrales en el desarrollo de los estudios urbanos locales, como César Vapñarsky y Mario Robirosa. Por otro lado, Vapñarsky figura entre la planta de profesores de la carrera en el año 1959. (Kóning, 2014) 
Además de los estudios relacionados con el rol de la ciudad en el desarrollo, Germani participa con el equipo Urbis de arquitectos para el Plan Urbano para Posadas en el año 1957 y realiza el trabajo sobre la Isla Maciel en la que participan algunos arquitectos, como Mario Robirosa, que tuvieron un rol importante en el desarrollo de los estudios urbanos. (Entrevista a Mario Robirosa, 2017)

En relación con la carrera de Sociología y sus programas de estudio, en 1973 se reformula el programa de estudios y se incorpora la materia "Sociología Urbana" como asignatura especial, además de "Planeamiento urbano y regional". En el programa de 1976 y en el de 1984 existe la asignatura Sociología Urbana como materia especial y así continúa hasta la actualidad (Mancuso, 2011). También existió de modo intermitente dentro de la carrera de Arquitectura, Diseño y Urbanismo de la UBA.

\section{La década de 1960 y la creación del Instituto de Planeamiento Regional y Urbano del Litoral (IPRUL). La sociología urbana en dicho contexto.}

En el año 1961 se crea en Rosario el Instituto de Planeamiento Regional y Urbano del Litoral (IPRUL) que funcionó entre 1962 y 1965, bajo la dirección del arquitecto Jorge Enrique Hardoy. Este centro reemplazó al Instituto de Arquitectura y Planeamiento de la Universidad del Litoral y su función fue generar estudios, investigaciones y planes reguladores elaborados bajo las nociones de planificación urbana y regional tributarias del desarrollismo y de la CEPAL, es decir, bajo el criterio de que la planificación estatal podía influir positivamente en el desarrollo equilibrado de los países. (Stropparo, 2011; Monti, 2013; Jajamovich y otros 2016)

Hardoy se graduó como Máster en Planificación Urbana y Regional en Harvard en 1955, una universidad de impronta desarrollista, en donde luego se doctoró en 1966. (Monti, 2013) Con ese criterio de planificación urbana formó un equipo integrado por profesionales de distintas disciplinas entre cuyos profesores figuraron Oscar Yujnovsky y Mario Robirosa. Más allá de que todos los profesionales son clave en la historia y consolidación de los estudios urbanos locales, en este escrito me centraré en el Arq Mario Robirosa porque es quien tuvo a cargo la materia "Sociología Urbana" en el IPRUL y porque todos sus discípulos lo reconocen como "sociólogo" aunque en verdad no tenía título de grado en esta disciplina.

Robirosa se graduó de arquitecto en 1957 y durante su carrera recibió cursos del cura belga Francois Houtart, sociólogo marxista, diplomado en urbanismo y con un perfil altamente comprometido con los sectores postergados de las sociedades y con el padre Lebret, francés, creador del concepto de "desarrollo integral" que fue una versión superadora de la perspectiva meramente economicista del desarrollo y que incluye la calidad de vida de toda la población, es decir, un concepto precursor del Desarrollo Sustentable. Lebret fue un impulsor de los estudios de planeamiento urbano y habitacional y fue referente para muchos investigadores argentinos tales como Jorge Enrique Hardoy, Ramón Gutiérrez, Floreal Forni, Ezequiel Ander-Egg y el mismo Robirosa, por sólo citar los relacionados con el área urbana y habitacional.

Una vez graduado Robirosa realizó estudios de postgrado en Francia durante los años '60 con el antropólogo Pierre Henri Chombart de Lauwe a quien se considera un iniciador de la sociología urbana en Francia. Chombart fue un estudioso de los sectores populares, la vida urbana y la relación de la clase obrera con la vivienda, que tiene entre sus referentes teóricos a Durkheim y la morfología urbana, a Marcel Mauss y a la Escuela de Chicago, aunque refuta varios de los postulados de esa Escuela sobre la desorganización familiar y la anomia y es un fuerte defensor de la cultura obrera. Fue director del Centre de Sociologie Urbaine creado por el estado para avalar su política de vivienda pero, al revés de lo esperado, sus estudios fueron muy críticos de la política pública en materia habitacional. Las técnicas usadas por Chombart aúnan la etnografía, las estadísticas y la foto área, técnica de la que fue precursor. (Ullán de la Rosa, 2014).

Durante su estadía en Francia, y bajo la dirección de Chombart, Robirosa toma cursos de antropología, psicología social, estadística y representación gráfica de fenómenos sociales y además participa en la investigación Famille et Habitation (1960) de Chombart, en donde estudian tres tipos de vivienda obrera construida por el estado y sus efectos sobre la vida y la cultura obrera. Entre ellos, la Cité Radiese de Nantes, proyectada por Le Corbusier, a la que Chombart critica profundamente por su esquema desintegrador del estilo de vida de la clase obrera y que se considera el primer trabajo crítico de la arquitectura funcionalista y racionalista tributaria de La Carta de Atenas (Ullán de la Rosa, 2014). Robirosa, en Francia, toma contacto, además, con el texto la "Ecología humana" de Amos Hawley que, como comenta él mismo, se 
convirtió en la base de su concepción de la sociología urbana durante años (Entrevista a Mario Robirosa, 2017). Sintéticamente, la ecología humana, es una derivación de la ecología urbana de Chicago que estudia las relaciones de los hombres con su ambiente tanto natural como construido y social. Estudia los esfuerzos adaptativos de los grupos humanos a esa diversidad de entornos y también los métodos para mejorar esa relación. (Hawley, 1975).

A su regreso en 1960 toma contacto nuevamente con Gino Germani y realiza el "certificado de estudios sociológicos" que dictaba el sociólogo italiano para los profesionales provenientes de otras disciplinas. En esa instancia conoce a Vapñarsky y Víctor Segal y reemplazan a Germani en el grupo Urbis.

En 1961 conoció al Arquitecto Jorge Enrique Hardoy y se incorpora al IPRUL que se sostenía con fondos de la Fundación Di Tella, la Fundación Ford y el CONICET. El otro docente del IPRUL era Oscar Yujnovsky y el resto eran docentes de la Universidad del Litoral.

Robirosa armó su programa de estudios de sociología urbana del IPRUL bajo la perspectiva teórica de la Ecología Humana, más todas las referencias teóricas que traía de Francia y el importante conocimiento de técnica estadística, lo que le permitía tomar datos estadísticos primarios, en un contexto donde el financiamiento era abultado y permitía a los investigadores desarrollar datos de este tipo a gran escala. (Entrevista a Robirosa, 2017)

Por el IPRUL se desarrollaron los planes reguladores de Paraná, Concordia, Rafaela y otras pequeñas localidades de la región. Tal como comenta el mismo Robirosa, eran investigaciones con gran conocimiento directo de las sociedades en cuestión, pero sin mucha intervención de la población y sin amparo o demanda gubernamental de dichos planes, lo cual dio lugar a fuertes críticas a este modo de planificar, incluso por parte del propio Robirosa que hizo de esa rama la "planificación estratégica" - el centro de su carrera profesional y docente.

En 1965 se suspenden las actividades del IPRUL por conflictos internos con la Universidad del Litoral. Jorge Enrique Hardoy reabrirá las actividades del Instituto con parte del equipo (Yujnovsky, Robirosa, Rofman, Basaldua y Luetich) creando el Centro de Estudios Urbanos y Regionales (CEUR) con sede en el rectorado de la Universidad de Buenos Aires. (Monti, 2013)

\section{El Centro de Estudios Urbanos y Regionales (CEUR), los estudios urbanos y la sociología.}

A principios de 1966 el anterior instituto reabre con el nombre de Centro de Estudios Urbanos y Regionales (CEUR) bajo dependencias de la UBA, pero a partir del golpe de estado de junio de 1966 se separa de la universidad para no tener que aceptar a las autoridades militares. Desde 1967 funcionó como centro asociado del Instituto Di Tella, hasta 1976, en que disminuye sus actividades por causa del golpe de estado de ese año.

En el CEUR trabajó el mismo equipo que funcionaba en Rosario y desde 1973 abre un Programa de Formación de Investigadores en Desarrollo Urbano y Regional que tuvo dos promociones: 1973-1974 y 1975-1976. Allí se formaron buena parte de los investigadores que conformaron el campo de los estudios urbanos locales a partir de la década del '60 hasta la actualidad.

EI CEUR tuvo dos líneas importantes de trabajo: regional rural y urbana. Dentro de los temas urbanos que se investigaron, se cuentan: la vivienda, la política pública en materia urbana, el rol del estado de la construcción de grandes obras públicas (CEUR, 2013)

Entre los sociólogos que estudiaron el postgrado del CEUR se cuentan Mabel Manzanal, Beatríz Schmukler y Alicia Ziccardi, esta última dedicada a temas urbanos. También fue profesor visitante el Lic Alberto Federico que estuvo a cargo de la asignatura Sociología Urbana de la carrera de Sociología de la UBA hasta bien entrados los años '90 y el Lic. Ernesto Pastrana que actúa como consejero de algunas tesis producidas en esa Institución. (CEUR, 2013)

En cuanto a las orientaciones teóricas de los estudios de esta etapa, resultan claras las palabras de Alicia Ziccardi en las memorias de los 50 años del CEUR:

... Era evidente que el intenso proceso de urbanización ya no podía vincularse de manera directa con un proceso de industrialización que mostraba claros signos de agotamiento y que era producto de las masivas migraciones campo-ciudad. Eran ya claros los límites de las propuestas de desarrollo autosostenido que dieron origen a las teoría de la dependencia y la marginalidad con que intentaban explicarse la presencia de una masa de trabajadores que no lograba insertarse plenamente en el proceso productivo pero que mostraba gran capacidad de movilización..."

“...Hacia finales de los años sesenta se consolidaron en diferentes países de América Latina grupos de investigadores dedicados al estudio de los problemas urbanos (...) que 
reorientaron la investigación latinoamericana con los desarrollos teóricos de la sociología francesa (...) especialmente "La cuestión urbana" de Manuel Castells..."(Ziccardi, 2013)

En estas palabras de Alicia Ziccardi queda claro el agotamiento teórico de la teoría del desarrollo para explicar el proceso de urbanización y el rol de las ciudades en la modernización de la ciudad. También quedan claros los nuevos marcos teóricos que tomarán fuerza hacia fines de la década de 1960: la teoría de la dependencia y el marxismo estructuralista francés, a partir de los cuales la pobreza urbana ya no es más un resabio de la integración social débil sino un el signo de una formación económico social dependiente que coloca a los pobres en ese lugar como parte de una dinámica económica y social general del país en relación con otros países.

\section{En síntesis:}

Si bien la reflexión sobre la ciudad y los problemas urbanos estuvo desde siempre en los pensadores que se consideran precursores del pensamiento sociológico (abogados, médicos), pueden situarse los inicios de la sociología urbana en Argentina con los escritos de Gino Germani dedicados a la ciudad y su rol en la modernización del país.

En un contexto histórico caracterizado por la expansión de los distritos anexos a la Ciudad de Buenos Aires y otras ciudades intermedias a partir de las expectativas laborales que generó el proceso de sustitución de importaciones (Calello, 2000) surgieron nucleos poblacionales formados por migrantes cuyas problemáticas fueron interpretadas a luz de la teoría de la modernización, encabezada a nivel local por Gino Germani. La mirada sociológica de este teórico sobre la urbanización no es lineal, sino más bien vacilante ya que en algunos escritos pone el acento en la desorganización familiar, la anomia y los males urbanos como el alcoholismo y la prostitución y en escritos posteriores analiza la ciudad y la vida urbana como un motor para el desarrollo geográfico y social de los países.

Si se toma en cuenta la tesis de Oscar Yujnovsky sobre la existencia de dos escenarios diferentes en torno la modernización; uno primero formado alrededor del Seminario sobre problemas de urbanización en América Latina de 1959 en donde la mirada sociológica sobre las villas es de "anomia" y "patología" y un segundo escenario hacia fines de la década de 1960 en donde predomina el enfoque de la "modernización" y la urbanización desaparece como fuente de problemas y es vista como variable independiente necesaria para el desarrollo, entonces es posible considerar que la variación en los trabajos de Germani se corresponden con este cambio de perspectiva sobre el rol de la ciudad y de la modernización.

Más allá de esto, debe destacarse el rol de Germani en los inicios de la sociología urbana porque junto con él se formaron varios arquitectos que son pilares de los estudios urbanos locales como Robirosa y Vapñarsky y porque en esa currícula surge la primera cátedra de vivienda dentro de la recién creada carrera de sociología.

En 1961 se forma el Instituto de Planeamiento Regional y Urbano del Litoral (IPRUL) bajo la dirección del Arq Jorge Enrique Hardoy que es una figura destacada en los estudios urbanos del país. En ese instituto el Arq. Mario Robirosa dictará la materia Sociología Urbana con el encuadre general de la Ecología Humana de Hawley (relación ambiente - sociedad) y en un marco político económico más amplio que entiende al planeamiento como motor del desarrollo, en concordancia con la mirada internacional sobre el tema.

Algunos planes directores elaborados por la etapa como por ejemplo el Plan de Rafaela (Santa Fe), llevan los signos de la Escuela de Ecología de Chicago, pero desde un punto vista crítico que refuta el crecimiento en círculos concéntricos tal como los planteaba Burgess en 1925. En este plan también hay huellas de las otras técnicas aprendidas por Robirosa en Francia, tal como el uso de datos estadísticos primarios y secundarios y la foto aérea.

Hasta esta época, la teoría del desarrollo y la modernización es la perspectiva teórica dominante en los estudios de la sociología urbana, en un contexto aún dominado por las migraciones internas y la expansión de asentamientos precarios.

Los años '70 no sólo verán nacer nuevos centros, como el CEUR, sino nuevos paradigmas para interpretar la realidad social y urbana. Centralmente la teoría de la dependencia y el marxismo estructuralista encarnado por Manuel Castells. Desde estos paradigmas, la existencia de pobres urbanos en la ciudad ya no es considerada un momento previo a la integración en las estructuras modernas de la sociedad, sino el signo de una estructura socioeconómica dependiente que los tiene en ese lugar como parte de su engranaje general y en la cual el rol del estado es la dotación de consumos colectivos necesarios para la reproducción ampliada del capitalismo. Esta época 
es rica, también, en estudios que analizan el rol de los movimientos sociales urbanos para enfrentar la lógica del capitalismo. Algunas tesis defendidas en el postgrado del CEUR, como la de Alicia Ziccardi sobre las políticas de vivienda y los movimientos urbanos, llevan los signos de estas nuevas perspectivas teóricas. (Ziccardi, 1977)

El golpe de estado de 1976 señala la desaceleración de las actividades del CEUR, el ocultamiento de las actividades y el exilio de varios de sus integrantes. También marca el eclipse del marxismo estructuralista y de la teoría de la dependencia, marcos teóricos que, al menos en la Argentina posterior a la recuperación democrática (1983), comenzarán a convivir con otras perspectivas analíticas como el papel de los poderes locales, la descentralización y la perspectiva del actor social.

\section{BIBLIOGRAFÍA CITADA}

BLANCO, A. (2006) Razón y Modernidad. Gino Germani y la sociología en Argentina. Buenos Aires: Siglo XXI

BUCAFFUSCA, S, SERULNICOFF, M y SOLARI, F. (2008). "Temperaturas de época: Gino Germani y la fundación de la carrera de Sociología". In González, Horacio (compilador); Historia Crítica de la Sociología Argentina, Buenos Aires: Colihue, pags. 329-352

CALELLO, T. (2000). "Breve caracterización histórica de la Región Metropolitana de Buenos Aires" en Sâo Paulo em Perspectiva Vol 14, N 4, diciembre. (Disponible en http://www.scielo.br/scielo.php?script=sci arttext\&pid=S0102-88392000000400005) (Consulta: $7 / 3 / 2018)$

CAPEL, H (1975). "La definición de lo urbano". Estudios Geográficos, n 138-139, febrero-mayo 1975, págs. 265-301.

CENTRO DE ESTUDIOS URBANOS Y REGIONALES (2011). CEUR Cincuenta años. (Disponible en http://www.ceur-conicet.gov.ar/ceur 50.php). (Consulta: 20/12/2017)

CUENYA, B (2004). "Las cuestiones centrales de la investigación en cada época" en "Lo Urbano. Una agenda abierta". In IV Jornadas de Sociología, Facultad de Ciencias Sociales, UBA.

DUHAU, E, CORONA, R y MORENO, F. (1991). "La sociología y la ciudad: Panorama y perspectivas de los estudios urbanos en los años ochenta". Sociológica, Año 6, N 15, enero abril. (Disponible en http://www.sociologicamexico.azc.uam.mx/index.php/Sociologica/article/view/888) (Consulta: 7/3/2018)

GERMANI, G. (1967). "La ciudad como mecanismo integrador". Revista Mexicana de Sociología, Vol. 29, No. 3 (Jul. - Sep), pags. 387-406.

GORELIK, A. (2008) "La aldea en la ciudad. Ecos urbanos de un debate antropológico". RMA. Antropología Social I, Revista del Museo de Antropología, Universidad Nacional de Córdoba, págs. 73-96.

JAJAMOVICH, G y otros. (2016). "Ciudad latinoamericana. Teorías, actores y conflictos". Urbana, Rev. Eletrônica Cent. Interdiscip. Estud. Cid. Campinas (SP) v.8, n.3 [14] pags. 01-07

HAWLEY, A. (1975). Teoría de la ecología humana. Madrid: Tecno.

KOENING, E. (2014). "La carrera de Sociología de la Universidad de Buenos Aires entre 1966 y 1974". In VIII Jornadas de Sociología de la UNLP, diciembre.

LEWIS, O. (1961). Antropología de la pobreza. Cinco Familias. México: Fondo de cultura Económica. 
MANCUSO, M. (2011). "La Carrera de Sociología de la UBA, su currícula a través de los años. 1958-2011". In IX Jornadas de Sociología. Facultad de Ciencias Sociales, Universidad de Buenos Aires, Buenos Aires.

MONTI, A.. (2013). "Una escuela, dos institutos. Hardoy + Hardoy en Rosario". Revista Registros, Año 9 (№10), págs. 24-37

MONTI, A. (2015). "La enseñanza de la planificación Urbana en la Argentina: Jorge Enrique Hardoy, del IPRUL al CEUR (1926-1976). Anuario IEHS 2014-2015, págs.177-195

MONTI, A. (2017). "La Revista SIAP como mecanismo para la consolidación del saber experto en América Latina”. Estudios del hábitat, Vol. 15 (1) JUNIO 2017

(Disponible en revistas.unlp.edu.ar/hábitat). (Consulta: 10/1/2018)

NOVICK, A. (2004). "Historias del urbanismo. Historias de la ciudad. Una revisión de la bibliografía". Crítica 137, Instituto de Arte Americano, FADU, UBA (Disponible en www.iaa.uba.ar) (10/1/2018)

PEREYRA, D. (2008). "Sociología e Investigación social en la obra de Ernesto Quesada". Políticas de la Memoria, Anuario de Investigación. CEDINSI, Buenos Aires: 192-202.

REDFIELD, R. (1947). "The folk society". The American Journal of Sociology v 52, N 4. Chicago: The University of Chicaggo Press.

ROFFE, P. (2013). "Modernización y urbanización: Las claves y condiciones del recorrido de la." In $X$ Jornadas de Sociología, Facultad de Ciencias Sociales, UBA.

SAUNDERS, P (1986). Social Theory and the Urban Question. Nueva York, Holmes and Meier.

STROPPARO, P. (2011). "Teorías del desarrollo en América Latina entre la década del cincuenta y la década del setenta del siglo XX". In IX Jornadas de Sociología. Facultad de Ciencias Sociales, Universidad de Buenos Aires, Buenos Aires, 2011 (Disponible en http://cdsa.aacademica.org/000-034/231.pdf) (Consulta:I 26/11/2017)

SCHTEINGART, M. (2000). "La investigación urbana en América Latina".Papeles de Población, vol 6, núm 23, enero-marzo. Universidad Autónoma del Estado de México, Toluca, México.

SOCOLOFF, I (2012). "De la "sociología de la ciudad" a la "sociología urbana argentina"-Algunos indicios sobre los vínculos entre la sociología y la ciudad en Argentina". Ensemble. Revista electrónica de la casa argentina en París, Volumen 4, , p.221-249

TOPALOV, C. (2012). "Treinta años de sociología urbana. Un punto de vista francés". Conferencia dictada por Christian Topalov en la Universidad de General Sarmiento, octubre de 2012. (Disponible en http://habitarargentina.blogspot.com.ar/2012/10/conferencia-christiantopalov-treinta.html) (Consulta: 10/1/2018)

TROVERO, J. (2017). "Gino Germani. Investigación empírica y sociología científica. Un abordaje teórico metodológico de los estudios sobre urbanización en la Argentina (1957-1958). Miríada, Año 9, N 13, págs. 221-249.

ULLÁN DE LA ROSA, F. (2014). Sociología Urbana: de Marx y Engels a las escuelas posmodernas. Madrid: Centro de Investigaciones Sociológicas.

YUJNOVSKY, O. (1975). "Notas sobre la investigación de la configuración espacial interna y las políticas de uso del suelo urbano en América Latina". Revista Interamericana de Planificación N 35. Sociedad interamericana de planificación, SIAP, págs 5-22.

ZICCARDI, A. (1977). Políticas de vivienda y movimientos urbanos. El caso de Buenos Aires (1963-1973). Informe final para el Programa de Formación de Investigadores en Desarrollo Urbano y Regional. 1975-1977. CEUR- Di Tella. 